\title{
A Modelica Library for Simulation of Elecric Energy Storages
}

\author{
M. Einhorn ${ }^{1}$ \\ F. V. Conte ${ }^{1}$ \\ C. $\mathrm{Kral}^{1}$ \\ C. Niklas ${ }^{1}$ \\ H. Popp ${ }^{1}$ \\ J. Fleig ${ }^{2}$ \\ ${ }^{1}$ AIT Austrian Institute of Technology \\ Mobility Department, Electric Drive Technologies \\ Giefinggasse 2, 1210 Vienna, Austria \\ markus.einhorn@ait.ac.at \\ ${ }^{2}$ Vienna University of Technology \\ Institute of Chemical Technologies and Analytics \\ Getreidemarkt 9/164ec 1060 Vienna, Austria
}

\begin{abstract}
This article gives an overview of the Electric Energy Storage (EES) library, which is proposed for inclusion in the Modelica Standard Library. The library contains models with different complexity for simulating of electric energy storages like batteries (single cells as well as stacks) interacting with loads, battery management systems and charging devices. It is shown how the models are defined and how they can be parametrized. Finally, two example simulations are presented.
\end{abstract}

Keywords: Energy storages, library, battery simulation

\section{Introduction}

Simulation is a commonly used technique to reduce costs during the design and development process. The energy storage system is a key issue, especially for electric vehicles. Basic models of electric energy storages (EES) are already included in the commercial SmartElectricDrives (SED) library [1]. The EES library, presented in this article, provides basic as well as more complex models for battery cells and for battery stacks. It includes models for battery monitoring and measurement, chargers, loads, sensors and battery management. This library can be used to simulate the behavior of electric energy storages in mobile devices, stationary applications and in transportation systems including hybrid as well as electric vehicles. The models of the EES library are designed as universally as possible so that even very specific scenarios can be simulated by varying the parametrization. In the future it is intended to include the EES library in

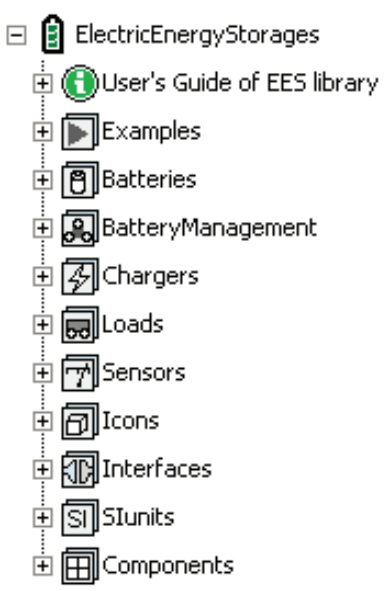

Figure 1: Electric Energy Stroage (EES) library structure

the Modelica Standard Library (MSL).

This article shows how the EES library is structured, how the fundamental models are defined and which parameters are needed. Finally, two example simulations are presented. For implementing the EES library, Dymola 7.4 and Modelica 3.2 are used [2-4].

\section{Library structure}

The EES library is structured as shown in Fig. 1 The fundamental packages and models are now explained in more detail.

\subsection{Batteries}

The Batteries package contains models for cells as well as for stacks with $n_{s}$ serially connected cells and $n_{p}$ cells in parallel. Its structure is shown in Fig. 2 


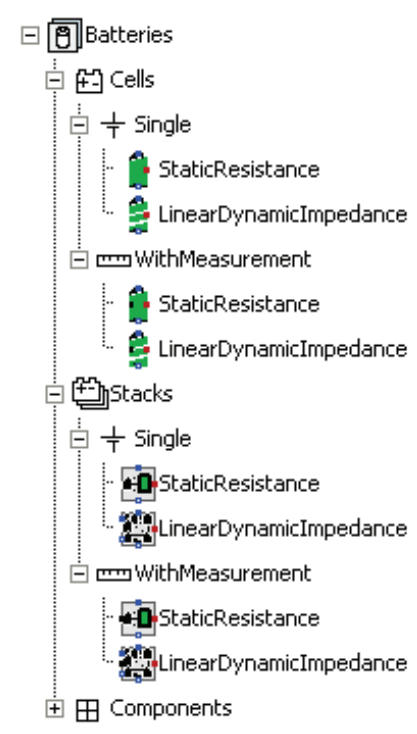

Figure 2: Structure of the Batteries package as a part of the EES library.

\subsubsection{Cells}

In the Cells package there are two different types of cell models: Single and WithMeasurement. While the Single cell models are models of the battery cell only, the WithMeasurement cell models extend the Single cell models with basic measurement. Each of these models can either be a simple cell model with just an ohmic impedance (StaticResistance) or a more complex cell model considering basic self discharge, a variable ohmic impedance and a variable number of variable RC elements (LinearDynamicImpedance).

\section{Single}

The StaticResistance single cell model as well as the LinearDynamicImpedance single model are shown in Fig. 3 and combined in the Single package.

Both single cell models have a positive (pin_p), a negative (pin_n) and an optional temperature connector (heatPort). If the heatPort is not used, it can be disabled and the model operates at a fixed temperature ( $\left.T_{\text {operational }}\right)$. The common parameters of the StaticResistance and the LinearDynamicImpedance cell models are given in Table1, For the StaticResistance single cell model additionally to Table 1, the parameter given in Table 2 is necessary. Instead, for the LinearDynamicImpedance single cell model, additionally to Table 1 the parameters given in Table 3 are necessary.
Table 1: Common input parameters of the StaticResistance and the LinearDynamic Impedance cell model.

\begin{tabular}{|c|c|c|}
\hline name & unit & description \\
\hline$S O C_{i n i}$ & & initial state of charge \\
\hline OCVtable & $\mathrm{V}$ & $\begin{array}{l}\text { lookup table for the open circuit } \\
\text { voltage } O C V \text { vs. the state of charge } \\
S O C\end{array}$ \\
\hline$t_{\text {total }}$ & $\mathrm{s}$ & total cell life time \\
\hline$Q_{\text {ini }}$ & $\mathrm{C}$ & initial transferred charge \\
\hline$Q_{\text {total }}$ & $\mathrm{C}$ & total transferable charge \\
\hline$C_{0}$ & $\mathrm{C}$ & $\begin{array}{l}\text { capacity at } T_{r e f} \text { for } Q_{a b s}=0 \text { and } \\
t=0\end{array}$ \\
\hline$k_{C t}$ & $\mathrm{C} / \mathrm{s}$ & $\begin{array}{l}\text { linear } t \text { dependency of the capacity } \\
C\end{array}$ \\
\hline$k_{C} Q_{a b s}$ & $\mathrm{C} / \mathrm{C}$ & $\begin{array}{l}\text { linear } Q_{a b s} \text { dependency of the ca- } \\
\text { pacity } C\end{array}$ \\
\hline$x_{C}$ & & $\begin{array}{l}\text { factor at which value of the capac- } \\
\text { ity } C S O H_{C}=0\end{array}$ \\
\hline useHeatPort & & $\begin{array}{l}\text { boolean variable for using the heat } \\
\text { port }\end{array}$ \\
\hline$T_{\text {operational }}$ & $\mathrm{K}$ & $\begin{array}{l}\text { operational temperature if the heat } \\
\text { port is not used }\end{array}$ \\
\hline$T_{\text {ref }}$ & K & reference temperature \\
\hline alphaRs & $\mathrm{K}^{-1}$ & linear temperature coefficient of $R_{s}$ \\
\hline alphaC & $\mathrm{K}^{-1}$ & $\begin{array}{l}\text { linear temperature coefficient of the } \\
\text { capacity } C\end{array}$ \\
\hline
\end{tabular}

Table 2: Additional input parameter of the StaticResistance cell model.

\begin{tabular}{lcl}
\hline name & unit & description \\
\hline$R_{\text {sref }}$ & $\Omega$ & $\begin{array}{l}\text { ohmic resistance at reference temperature } \\
T_{\text {ref }}\end{array}$ \\
\hline
\end{tabular}

The output variables both for the StaticResistance single cell model and the LinearDynamicImpedance are given in Table 4 and the calculation of them are presented in the following.

Starting from $S O C_{i n i}$ the

$$
S O C=S O C_{i n i}-\frac{Q}{C},
$$

with the removed charge

$$
Q=\int_{t_{\text {start }}}^{t_{\text {stop }}} I(t) d t
$$

The open circuit voltage $O C V$ of a battery cell changes with $S O C$ and can be extracted from a lookup table OCVtable between the charging voltage limit $C V L$ and the discharging voltage limit $D V L$. This linearly interpolated lookup table for a lithium ion ( $\mathrm{Li}-$ ion) battery cell [5] is exemplarily shown in Fig. 4] 


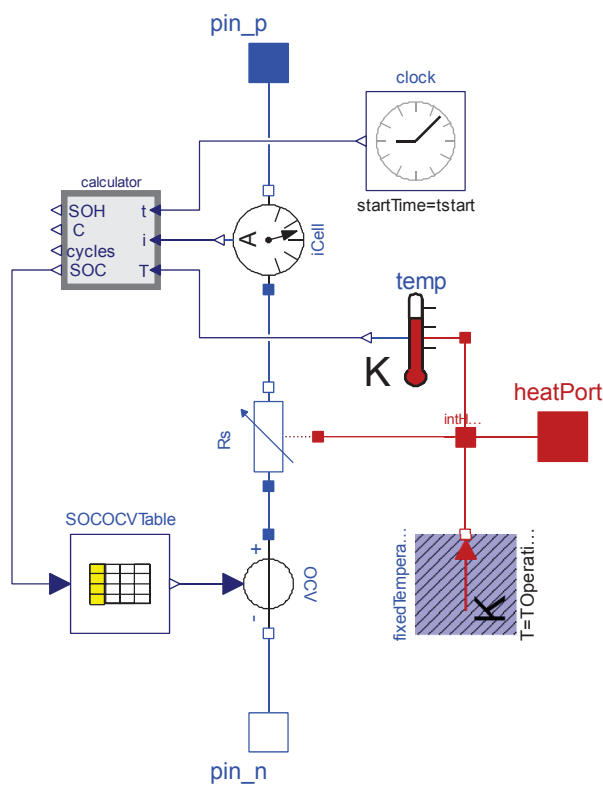

(a) StaticResistance

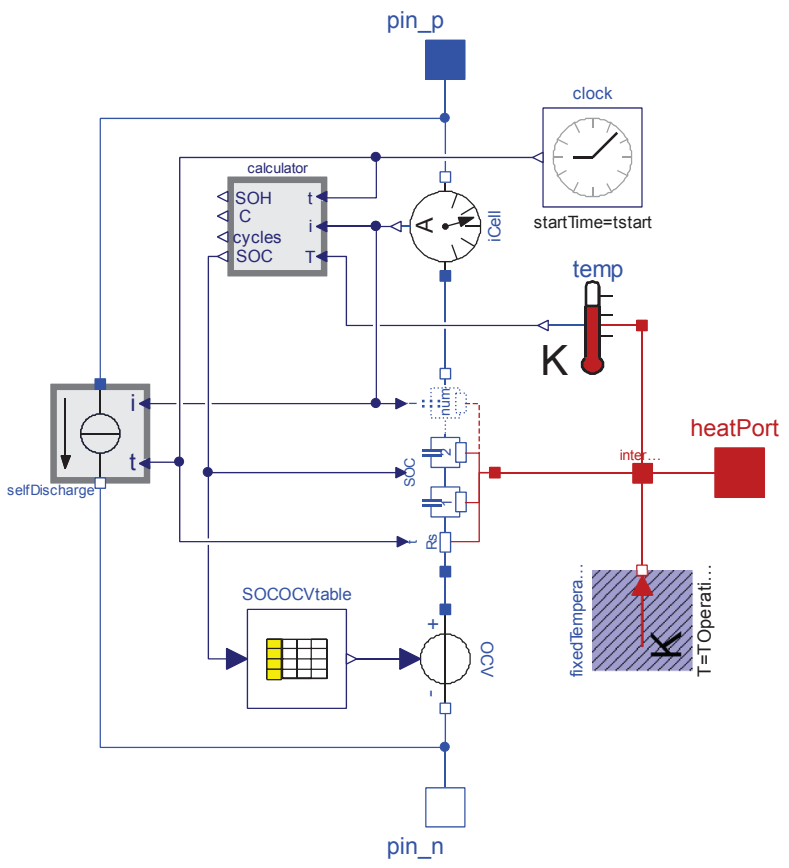

(b) LinearDynamic Impedance

Figure 3: Single cell model with a static impedance (a) and with a variable number of variable RC elements, a variable ohmic impedance as well as with basic self discharge (b)

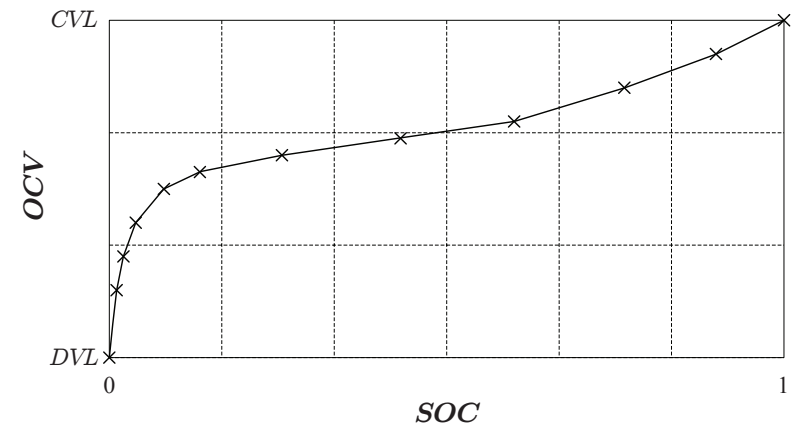

Figure 4: Linear interpolation of the measured open circuit voltage $(O C V)$ for different state of charge $(S O C)$ of a Li-ion battery cell [5].

The total transferred charge between $t_{\text {start }}$ and $t_{\text {stop }}$ is

$$
Q_{a b s}=Q_{\text {ini }}+\int_{t_{\text {start }}}^{t_{\text {stop }}}|I(t)| d t
$$

and the equivalent number of cycles

$$
\text { cycles }=\text { cycles }_{\text {ini }}+\int_{t_{\text {start }}}^{t_{\text {stop }}} \frac{|I(t)|}{2 \cdot C} d t,
$$

with

$$
\text { cycles }_{\text {ini }}=\frac{Q_{\text {ini }}}{2 \cdot \frac{C_{0}+C\left(t_{\text {start }}, Q_{\text {ini }}, T_{\text {heat } P \text { ort }}\right)}{2}},
$$

relates the total transferred charge to the cell capacity $C$. Therefore, one cycle is equivalent to the charge transfer (regardless in which direction) of one full discharge and one full charge of the current capacity $C$.

Both cell models consider basic aging, which can be divided into calendaric aging and aging due to cycling. Calendaric aging of a cell is estimated from the time $t$ and the absolute transferred charge $Q_{a b s}$ defines the aging due to cycling. Aging of a battery mainly influences the capacity $C$ (decreasing) and the internal impedance (increasing).

The cell capacity

$$
\begin{aligned}
C=\left(C_{0}+k_{C t} \cdot t+k_{C} Q_{a b s} \cdot Q_{a b s}\right) \\
\cdot\left(1+\text { alphaC } \cdot\left(T_{\text {heatPort }}-T_{\text {ref }}\right)\right)
\end{aligned}
$$

is temperature dependent and decreases with increasing time $t$ (calendaric aging) as well as with increasing transferred charge $Q_{a b s}$ (aging due to cycling).

The difference between the StaticResistance and the LinearDynamicImpedance single cell model is the configuration of internal impedance on the one hand and the self discharge on the other hand.

The StaticResistance single cell model has just a single, temperature dependent, ohmic 
Table 3: Additional input parameters of the LinearDynamicImpedance cell model.

\begin{tabular}{|c|c|c|}
\hline name & unit & description \\
\hline$I_{s d 0}$ & A & $\begin{array}{l}\text { self discharge current at } T_{r e f} \text { for } \\
Q_{a b s}=0 \text { and } t=0\end{array}$ \\
\hline$k_{s d t}$ & $\mathrm{~A} / \mathrm{s}$ & $\begin{array}{l}\text { linear } t \text { dependency of self dis- } \\
\text { charge current }\end{array}$ \\
\hline$k_{s d} Q_{a b s}$ & $\mathrm{~A} / \mathrm{C}$ & $\begin{array}{l}\text { linear } Q_{a b s} \text { dependency of self dis- } \\
\text { charge current }\end{array}$ \\
\hline alphasd & $\mathrm{K}^{-1}$ & $\begin{array}{l}\text { linear temperature coefficient of } \\
\text { self discharge current } I_{s d}\end{array}$ \\
\hline$R_{s 0}$ & $\Omega$ & $\begin{array}{l}\text { series resistance at } T_{r e f} \text { for } \\
Q_{a b s}=0 \text { and } t=0\end{array}$ \\
\hline$k_{R_{s} S O C}$ & $\Omega$ & linear $S O C$ dependency of $R s$ \\
\hline$k_{R_{s} t}$ & $\Omega / \mathrm{C}$ & linear $t$ dependency of $R s$ \\
\hline $\begin{array}{l}k_{R_{s} Q_{a b s}} \\
\text { num }\end{array}$ & $\Omega / \mathrm{C}$ & $\begin{array}{l}\text { linear } Q_{a b s} \text { dependency of } R s \\
\text { number of series RC elements }\end{array}$ \\
\hline$<R_{d 0}>$ & $\Omega$ & $\begin{array}{l}\text { array of length num of } R_{d} \text { at } T_{r e f} \\
\text { for } S O C=0, Q_{a b s}=0 \text { and } t=0\end{array}$ \\
\hline$<k_{R_{d} S O C}>$ & $\Omega$ & $\begin{array}{l}\text { array of length num of linear } S O C \\
\text { dependency of } R d\end{array}$ \\
\hline$<k_{R_{d} t}>$ & $\Omega / \mathrm{s}$ & $\begin{array}{l}\text { array of length num of linear } t \text { de- } \\
\text { pendency of } R d\end{array}$ \\
\hline$<k_{R_{d} Q_{a b s}}>$ & $\Omega / \mathrm{C}$ & $\begin{array}{l}\text { array of length num of linear } Q_{a b s} \\
\text { dependency of } R d\end{array}$ \\
\hline$<$ alphaRd $>$ & $\mathrm{K}^{-1}$ & $\begin{array}{l}\text { array of length num of linear tem- } \\
\text { perature coefficient of } R_{d}\end{array}$ \\
\hline$<C_{d 0}>$ & $\mathrm{F}$ & $\begin{array}{l}\text { array of length num of } C_{d} \text { for } \\
S O C=0, Q_{a b s}=0 \text { and } t=0\end{array}$ \\
\hline$<k_{C_{d} S O C}>$ & $\mathrm{F}$ & $\begin{array}{l}\text { array of length num of linear } S O C \\
\text { dependency of } C d\end{array}$ \\
\hline$<k_{C_{d} t}>$ & $\mathrm{F} / \mathrm{s}$ & $\begin{array}{l}\text { array of length num of linear } t \text { de- } \\
\text { pendency of } C d\end{array}$ \\
\hline$<k_{C_{d} Q_{a b s}}>$ & $\mathrm{F} / \mathrm{C}$ & $\begin{array}{l}\text { array of length num of linear } Q_{a b s} \\
\text { dependency of } C d\end{array}$ \\
\hline$x_{Z}$ & & $\begin{array}{l}\text { factor at which value of the internal, } \\
\text { ohmic impedance } Z S O C_{Z}=0\end{array}$ \\
\hline
\end{tabular}

impedance, modeled as

$$
\begin{aligned}
& R_{s}= \\
& \quad R_{\text {sref }} \cdot\left(1+\text { alphaRs } \cdot\left(T_{\text {heatPort }}-T_{\text {ref }}\right)\right) .
\end{aligned}
$$

It does not consider impedance increase due to aging.

In contrast, the LinearDynamicImpedance single cell model has an ohmic impedance and num serially connected RC elements for the transient behavior of the electrodes of an electrochemical energy storage as shown in Fig. 5] [6, 7]. All ohmic impedances $\left(R_{s}, R_{d 1} \ldots R_{d \text { num }}\right)$ are temperature dependent and have a linear dependency on state of charge $S O C$, on the time $t$ (calendaric aging) as well as on the transferred charge $Q_{a b s}$ (aging due to cy-
Table 4: Calculated output variables of the StaticResistance and the LinearDynamic Impedance cell model.

\begin{tabular}{lll}
\hline name & unit & description \\
\hline$S O C$ & & state of charge \\
$O C V$ & $\mathrm{~V}$ & open circuit voltage \\
$Q_{a b s}$ & $\mathrm{C}$ & total transferred charge \\
cycles & & number of equivalent cycles \\
$t$ & $\mathrm{~s}$ & calendaric cell time \\
$S O H$ & & state of health \\
$S O S$ & & state of sickness \\
$C$ & $\mathrm{C}$ & capacity \\
$V$ & $\mathrm{~V}$ & cell voltage \\
\hline
\end{tabular}

cling). The serial resistor $R_{s}$ is modeled as

$$
\begin{aligned}
& \quad R_{s}= \\
& \begin{aligned}
\left(R_{s 0}+k_{R_{s} S O C} \cdot S O C+k_{R_{s} t} \cdot t+k_{R_{s} Q_{a b s}} \cdot Q_{a b s}\right) \\
\quad \cdot\left(1+\text { alphaRs } \cdot\left(T_{\text {heatPort }}-T_{\text {ref }}\right)\right) . \quad(8)
\end{aligned}
\end{aligned}
$$

For $n=1 \ldots$ num

$$
\begin{aligned}
& R_{d}[n]=\left(R_{d 0}[n]+\right. \\
& \left.k_{R_{d} S O C}[n] \cdot S O C+k_{R_{d} t}[n] \cdot t+k_{R_{d} Q_{a b s}} \cdot Q_{a b s}\right) \\
& \quad \cdot\left(1+\text { alphaRd }[n] \cdot\left(T_{\text {heat Port }}-T_{\text {ref }}\right)\right) .
\end{aligned}
$$

and since there is no temperature dependency for capacitances considered

$$
\begin{aligned}
C_{d}[n]= & C_{d 0}[n]+k_{C_{d} S O C}[n] \cdot S O C \\
& +k_{C_{d} t}[n] \cdot t+k_{C_{d} Q_{a b s}}[n] \cdot Q_{a b s} .
\end{aligned}
$$

Moreover, the LinearDynamic Impedance single cell model considers basic self discharge which is linear dependent on the temperature, the time (calendaric aging) and the transferred charge (aging due to cycling).

While with the StaticResistance single cell model only the basic impedance behavior can be simulated, the LinearDynamicImpedance can be used to simulate single cells very accurate if the parametrization work is well done.

The capacity as well as the internal impedance (only with the LinearDynamicImpedance single cell model) can change due to aging and the state of health $S O H$ (and accordingly the state of sickness $S O S$ ) compares the current condition of a battery cell to its ideal (initial) condition. The $S O H$ is divided into the $\mathrm{SOH}_{\mathrm{C}}$ and $\mathrm{SOH}_{Z}$ :

$$
S O H=1-S O S=S O H_{C} \cdot S O H_{Z},
$$




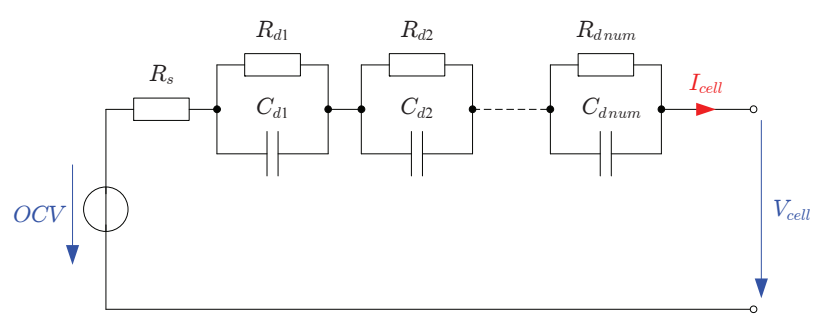

Figure 5: Battery model with one ohmic impedance and num serially connected RC elements.

as shown in Fig. 6

For a new cell, $S O H_{C}=1$ and $S O H_{Z}=1$ and therefore also $S O H=1$. When the capacity $C$ decreases (due to calendaric aging or aging due to cycling) to $x_{C} \cdot C_{0}$ (e.g. with $\left.x_{C}=0.8\right), S O H_{C}=0$. Hence,

$$
S O H_{C}=\frac{1}{C_{0} \cdot\left(1-x_{C}\right)} \cdot C-\frac{x_{C}}{1-x_{C}} .
$$

Similarly, when the sum of all internal, ohmic impedances

$$
Z=R_{s}+R_{d}[1]+R_{d}[2]+\ldots+R_{d}[\text { num }]
$$

increases (due to calendaric aging or aging due to cycling) to $x_{Z} \cdot Z_{0}$ (e.g. with $x_{Z}=2$ ), where

$$
Z_{0}=R_{s 0}+R_{d 0}[1]+R_{d 0}[2]+\ldots+R_{d 0}[\text { num }]
$$

$\mathrm{SOH}_{Z}=0$. Therefore,

$$
S O H_{Z}=\frac{1}{Z_{0} \cdot\left(1-x_{Z}\right)} \cdot Z-\frac{x_{Z}}{1-x_{Z}} .
$$

Fig.7 shows the dependency of $\mathrm{SOH}_{\mathrm{C}}$ and $\mathrm{SOH}_{Z}$ from $C$ and $Z$, respectively.

\section{WithMeasurement}

In the WithMeasurement package there are two cell models with measurement: The staticResistance and the LinearDynamicImpedance. Both contain basic measurement and instances of the corresponding single cell model. Fig. 8 shows for example the LinearDynamicImpedance with measurement. The voltage, the current and the temperature of the single cell model are measured and provided with the singleCellBus (cf. section 2.7). The cell models with measurement have the same connectors as the single cell models (pin_p, pin_n and heatPort) but have additionally the singleCellBus. Therefore several instances of these can be connected

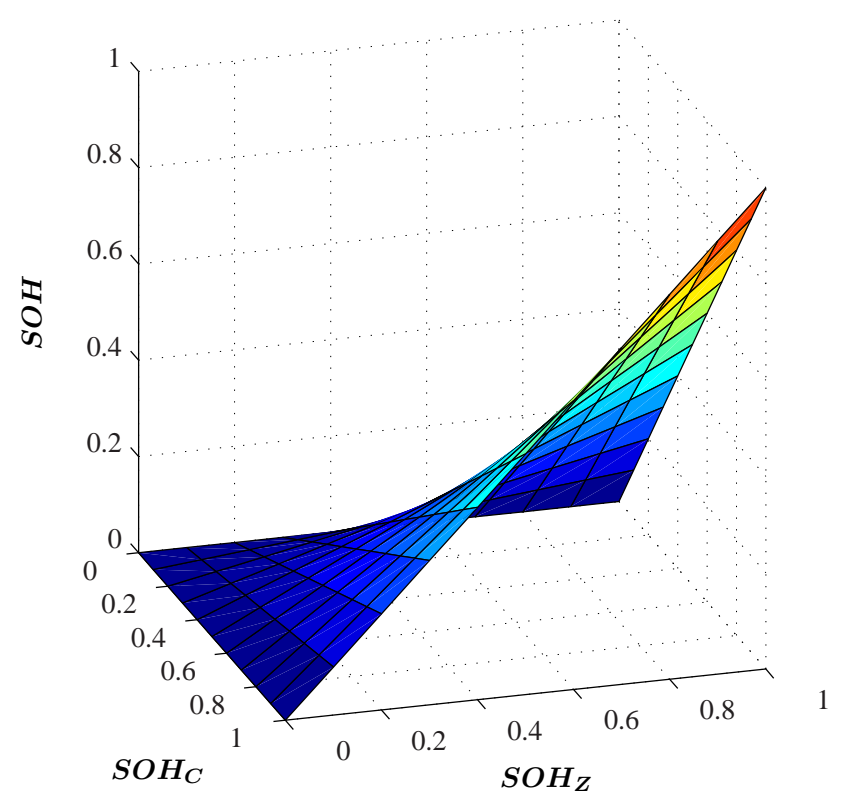

Figure 6: Dependancy of the state of health $\mathrm{SOH}$ from $\mathrm{SOH}_{\mathrm{C}}$ and $\mathrm{SOH}_{Z}$.

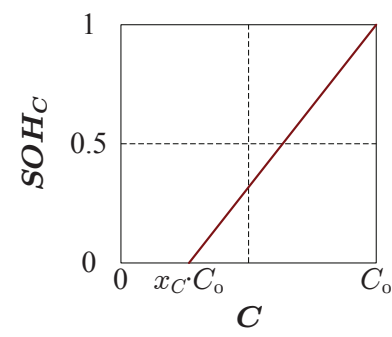

(a) $\mathrm{SOH}_{\mathrm{C}}$

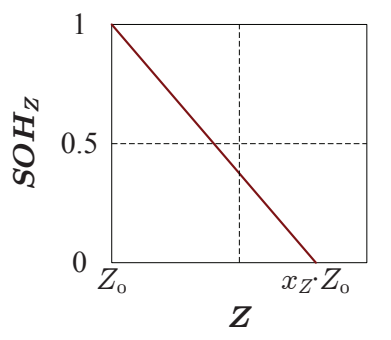

(b) $\mathrm{SOH}_{Z}$
Figure 7: Partial state of health with respect to the capacity $C$ and to the impedance $Z$ with paramters $0<x_{C}<1$ and $x_{Z}>1$.

together (serially and in parallel) and the basic measurement of each cell is done.

The advantage of separating the cell models in Single and WithMeasurement models shows up when it comes to stacks and the current of each cell should be measured. Fig. 9 shows the icons for the Single and WithMeasurement for the StaticResistance cell as well as for the LinearDynamic Impedance cell models with enabled heatPort.

\subsubsection{Stacks}

Stacks are $n_{s}$ serially connected cells and $n_{p}$ cells in parallel as shown in Fig. 10. The Stacks package is structured in the same way as the Cells package. There are Single and WithMeasurement stacks where each uses either the StaticResistance or 


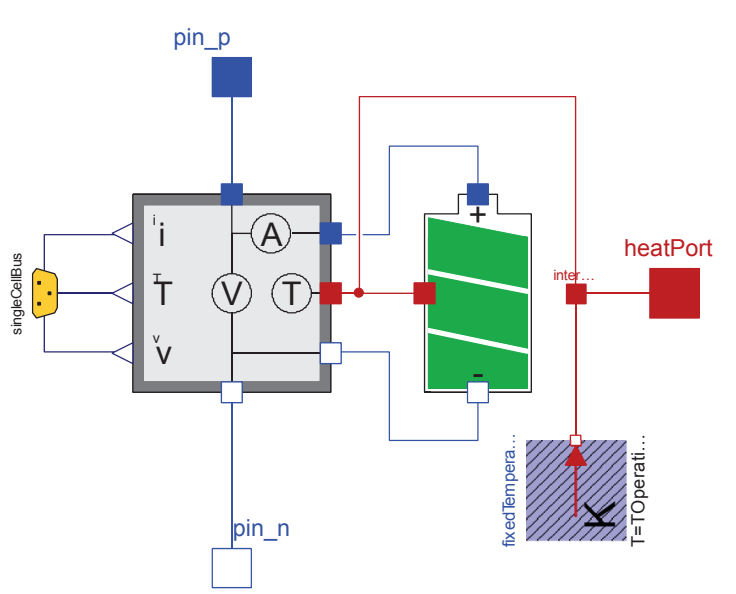

Figure 8: Cell model with measurement. The measured current, temperature and voltage are provided to the outside via the singleCellBus

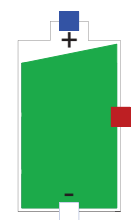

(a)

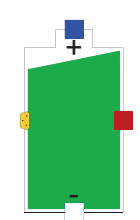

(b)

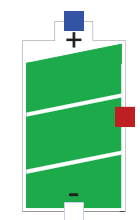

(c)

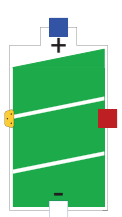

(d)
Figure 9: Icons for the StaticResistance (Single (a), WithMeasurement (b)) and for the LiniearDynamicImpedance (Single (c), WithMeasurement (d)) cell model with enabled heat port.

the LinearDynamicImpedance cell models.

The StaticResistance single stack model is a stack with all equal cells and therefore only one cell needs to be calculated and parameterized. It is basically a StaticResistance single cell model that scales the parameters of the model components and behaves like $n_{s} \cdot n_{p}$ equally parameterized instances of StaticResistance single cell models. For example the value of the series resistance is then $R_{s 0} \cdot n_{s} / n_{p}$ with the value $R_{s 0}$ for a single cell. Hence, it is much faster than a model with $n_{s} \cdot n_{p}$ instances of equally parametrized cell models.

The LinearDynamicImpedance single stack model has $n_{s} \cdot n_{p}$ instances of the LinearDynamicImpedance single cell model and the serial and parallel connections are textually generated with loops:

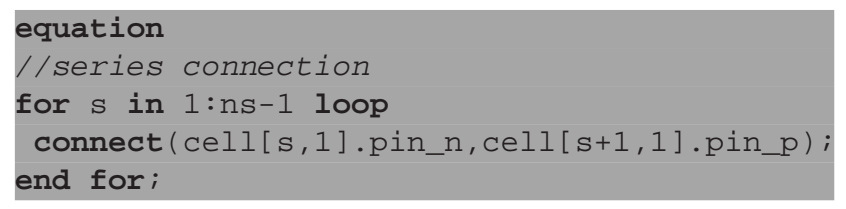

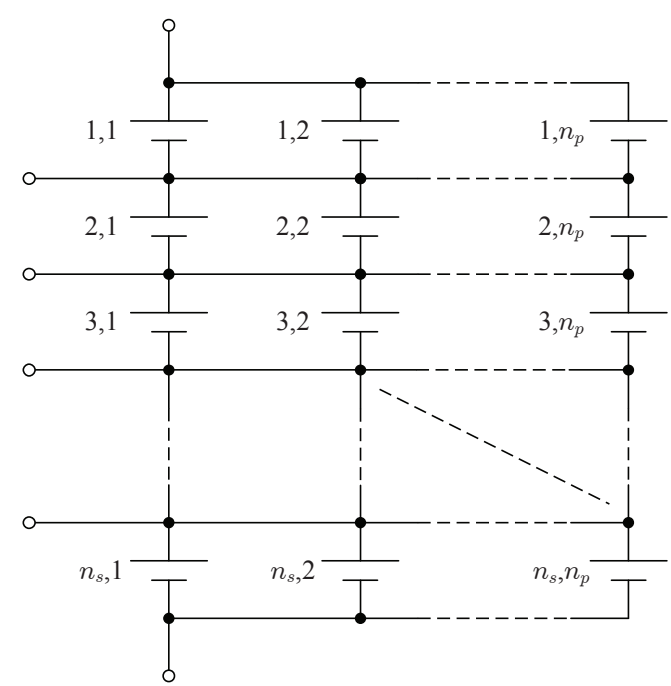

Figure 10: Battery stack with $n_{s}$ serially and $n_{p}$ connected cells.

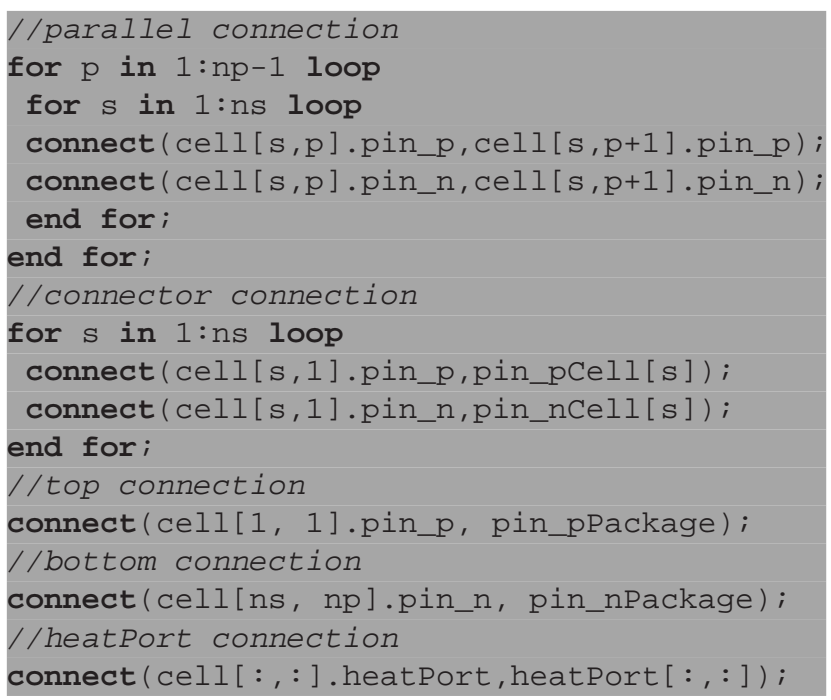

Each scalar parameter of the LinearDynamicImpedance single cell model is extended to an array of dimension $n_{s} \times n_{p}$ and the array parameters of the LinearDynamicImpedance single cell model now have the dimension $n_{s} \times n_{p} \times$ num. Each cell in this stack can be parametrized individually and therefore also cell variance in a stack can be simulated. All single cell connectors and all temperature connector are conditionally available. Fig. 11 shows the icons for all different stack models. Therefore a separate thermal model can be considered. With the single cell connectors and an additional model even cell balancing can be simulated [8].

From each single stack model (StaticResistance and LinearDynamicImpedance) there is also a version with measurement (StaticResistance 


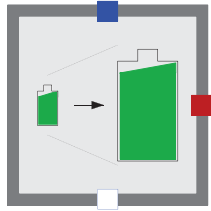

(a)

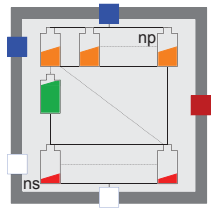

(c)

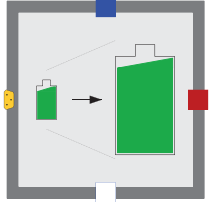

(b)

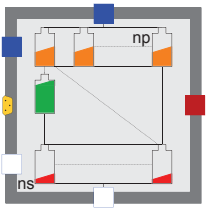

(d)
Figure 11: Icons for the Single (a), WithMeasurement (b) StaticResistance stack models with enabled heat ports and the Single (c), WithMeasurement (d) LinearDynamicImpedance stack models with enabled heat as well as single cell ports .

Table 5: Parameters of the Voltagecycling battery management model.

\begin{tabular}{lll}
\hline name & unit & description \\
\hline$n_{s}$ & & number of serially connected cells \\
$n_{p}$ & & number of parallel connected cells \\
$I_{\text {final }}$ & $\mathrm{A}$ & final charging switch off current \\
$V_{\text {max }}$ & $\mathrm{V}$ & maximal cell voltage \\
$V_{\text {min }}$ & $\mathrm{V}$ & minimal cell voltage \\
$t_{c}$ & $\mathrm{~s}$ & delay time after charging \\
$t_{d}$ & $\mathrm{~s}$ & delay time after discharging \\
$i n i$ & & true for initial discharging \\
\hline
\end{tabular}

and LinearDynamicImpedance) in the Stacks package.

\subsection{Battery Management}

In the current version of the EES library there is a voltage cycling device (VoltageCycling) implemented. Fig. 12 gives an overview about the VoltageCycling model and its parameters are given in Table 5

It basically has two boolean outputs to operate with loads and charging devices (cf. section 2.3 and 2.4). The cellBus can either be connected to a cell or a stack. During charging if $V_{\max }$ of any cell is reached and the charging current of all parallel connected cells is below $I_{\text {final }}$, the boolean output discharging gets true after $t_{c}$. When $V_{\min }$ of any cell is reached during discharging, the boolean output charging gets true after $t_{d}$. Therefore it is possible to cycle a cell or a stack within its voltage limits $V_{\min }$ and $V_{\max }$

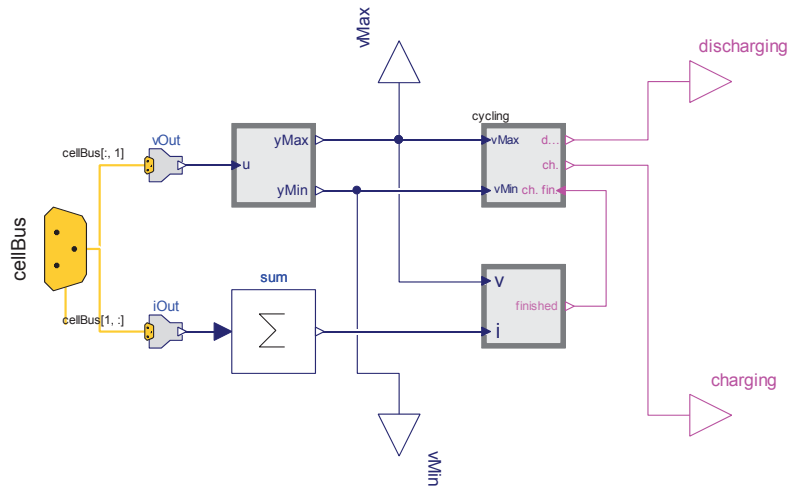

Figure 12: VoltageCycling model in the BatteryManagement package.

(in combination with a charging device and a load).

\subsection{Chargers}

In the Chargers package there is a constant current, constant voltage (CCCV) charging device modeled. A $\mathrm{CCCV}$ charging device charges a battery with a constant current until its $C V L$ is reached (constant current phase). Then a constant voltage is applied and the current decreases (constant voltage phase). The charging device is switched off when the charging current reaches the final charging switch off current (typically $5 \%$ of the $1 \mathrm{~h}$ discharging current).

When the boolean input of the modeled charging device is true (e.g. the boolean charging output charging from the VoltageCycling model described in section 2.2), a current source provides a constant current. This current is controlled by a voltage input (e.g. the maximal cell voltage in a battery stack), a reference voltage (parameter $V_{\max }$ ) and limited by the maximal charging current (parameter $I_{\max }$ ).

\subsection{Loads}

In the Loads package there are models to discharge a cell or a stack to cover typical lab situations (for example discharging a battery with a defined current or power profile). In extension to the electrical current sources (a current source parametrized with a negative current can be used as a load) there are four different loads considered:

- BooleanExternalControlledLoad

- BooleanConstantCurrent

- BooleanConstantPower 


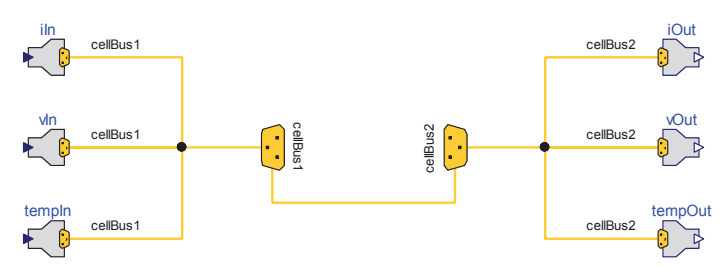

(a) CellBus

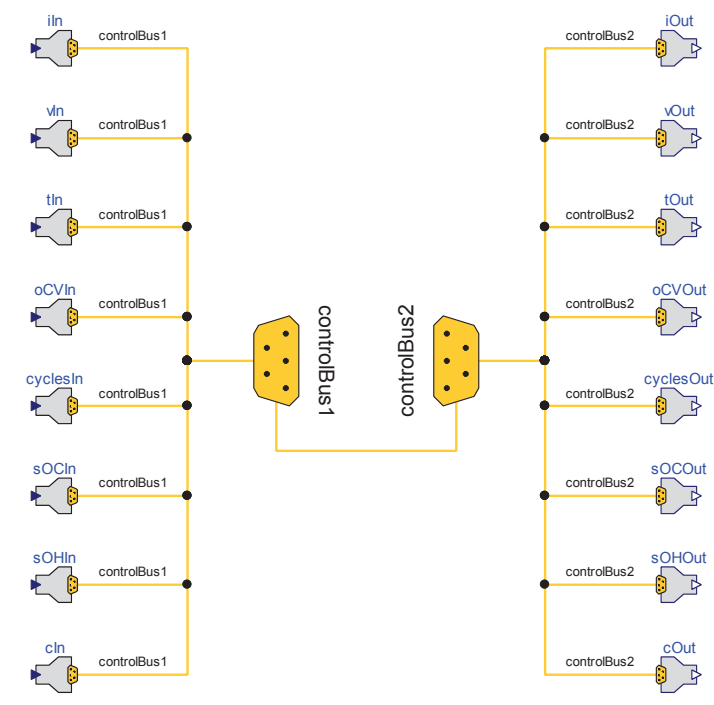

(b) ControlBus

Figure 13: Structure of the CellBus (a) and the ControlBus (b) with bus adaptors to all variables on the bus $(v, i, T$ for the CellBus and $v, i, T, O C V$, $S O C, S O H$, cycles, $C$ for the ControlBus).

\section{- Signalpower}

All Boolean models have a boolean input on which for example can be connected to the boolean output discharging of the VoltageCycling model (cf. section 2.2). If on is false the cell or the stack is not being discharged.

\subsection{Sensors}

The Sensors package provides models to estimate the energy, the charge and the absolute charge from/to a cell or a stack. The models provided in the Electrical.Analog. Sensors package from the MSL are used and consequently extended.

\subsection{Icons}

In this package there are combined all icons used for the packages.

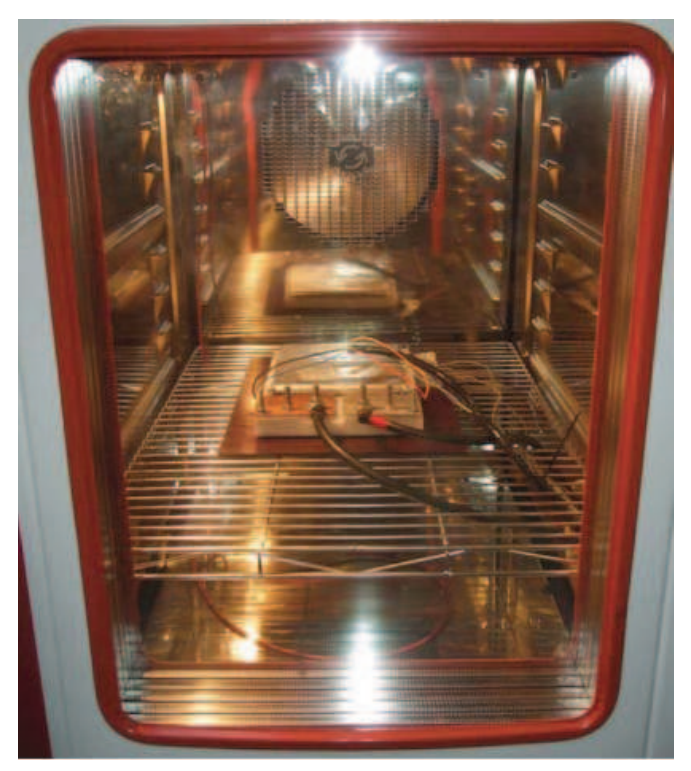

Figure 14: Test cell at constant temperature in a climate chamber.

\subsection{Interfaces}

There are two buses available: The CellBus and the ControlBus. The CellBus contains only measurable variables such as the cell voltage $v$, the cell current $i$ and the cell temperature $T$. It can be used when lab situations are simulated and only measurable variables are significant. It can also be used to test different battery monitoring systems, based on $i, v$ and $T$. The ControlBus contains the variables from the CellBus and additional the following: $O C V, S O C$, $S O H$, cycles and $C$. It could for example be the communication between the battery management system and the control units in an electric vehicle simulation.

In the Interfaces package there are also bus adapters to extract/inject all these variables (Real values) from/to the CellBus as well as from/to the ControlBus. Figure 13 shows the structure of the CellBus and of the ControlBus with the usage of all bus adapters.

\section{Examples}

Two different examples are presented:

First a LinearDynamicImpedance single cell model with one (num $=1$ ) RC element is parameterized according to [9]. A realistic current profile, gained from the FTP72 cycle is continuously applied to the parameterized model as well as to the real cell [10]. The FTP72 cycle is a standardized real life driving cycle that simulates an urban route of $12.07 \mathrm{~km}$. 

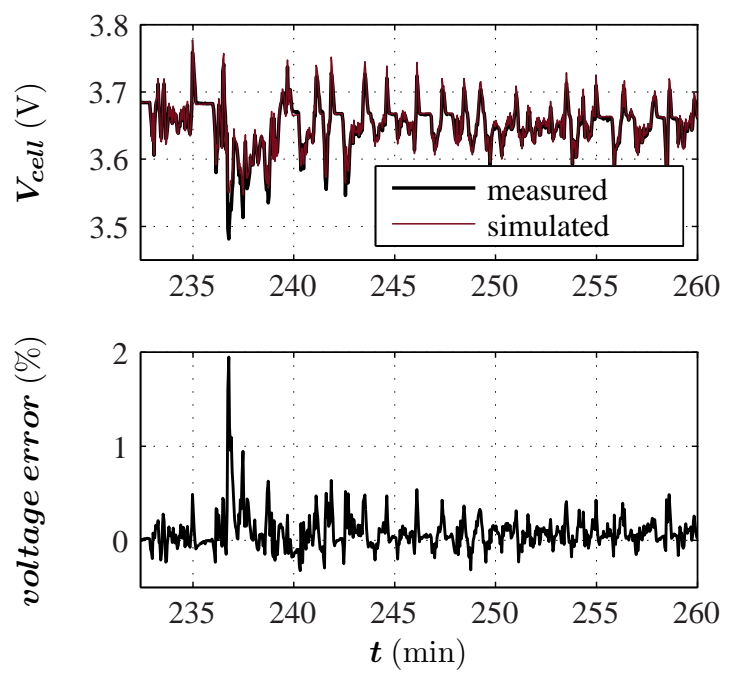

Figure 15: Comparison of the measured and simulated cell voltage when applying the FTP72 current profile to the real cell and to parameterized model (top). The error refers to the measured voltage (bottom).

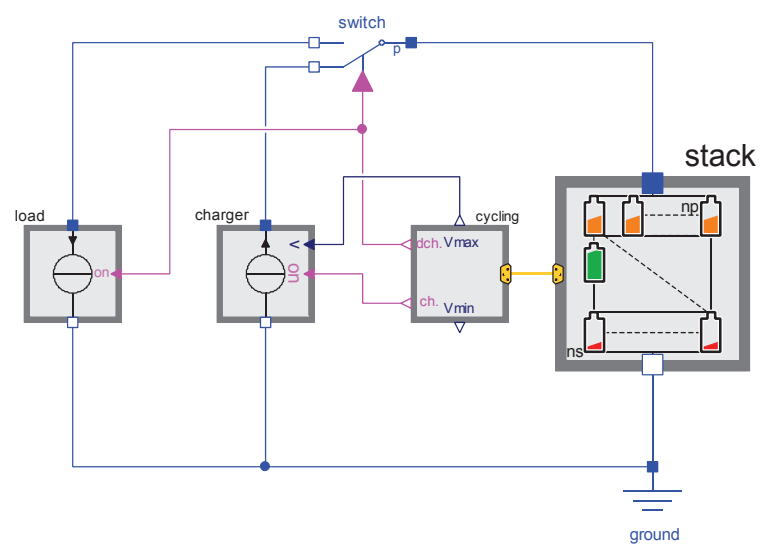

Figure 16: Simulation arrangement for cycling a battery stack.

This cycle is applied to a compact electric vehicle to extract the realistic current profile. The current profile is repeated starting from the full charged cell until it reaches discharging voltage limit $D V L$. This experiment is performed at constant temperature and Fig. 14 shows the test cell in a climate chamber. Fig. 15. compares the simulated to the measured cell voltage. The error, related to the measured voltage, between the simulated and the measured voltage stays below $2 \%$ at $S O C \approx 0.5$ during one complete FTP72 cycle. Therefore the chosen model approach is appropriate.

For the second example, three serially connected cells using the LinearDynamic Impedance stack model (without temperature and cell connectors) with different capacities are simulated. The cell capacities are $C_{1}=35 \mathrm{Ah}, C_{2}=40 \mathrm{Ah}, C_{3}=45 \mathrm{Ah}$ and
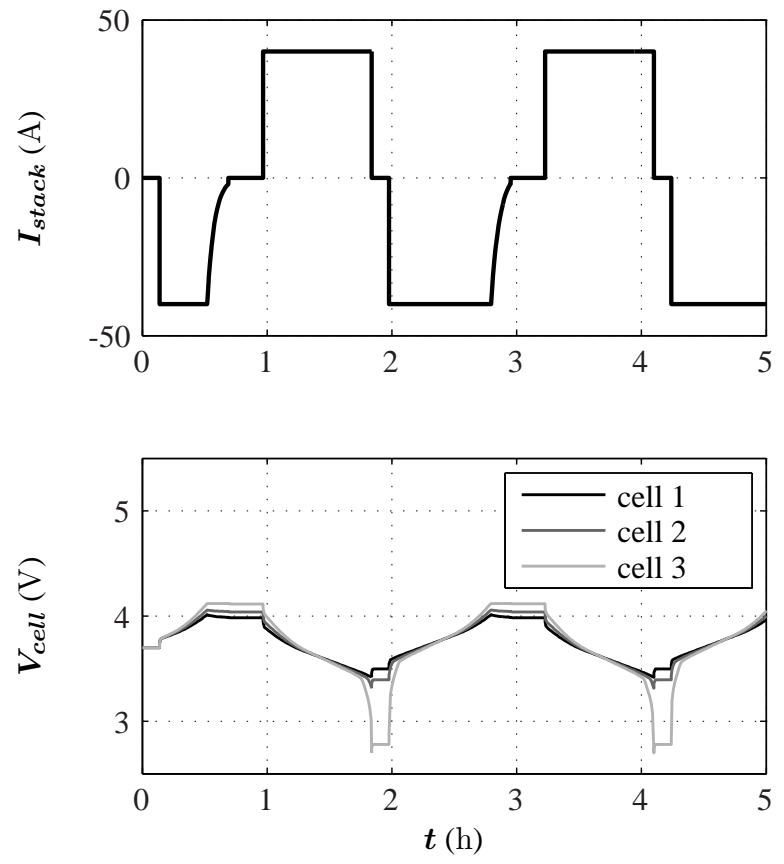

Figure 17: Current profile and voltage response from the three cells from the example simulation.

parametrized using [5, 9]. The cells are cycled with a $40 \mathrm{~A}$ charging and a $40 \mathrm{~A}$ discharging current between $C V L=4.1 \mathrm{~V}$ and $D V L=2.7 \mathrm{~V}$ with a delay after charginging $t_{c}=100 \mathrm{~s}$ and after discharging $t_{d}=50 \mathrm{~s}$. Cycling means continuously charging and discharging, which is a typical lab application for batteries. Fig. 16 shows the simulation arrangement with the charging device (charger), the battery management (cycling), the battery stack (stack), the discharger (load) and a switch (switch). Fig. 17 shows the battery stack current and the voltage from all three cells.

\section{Conclusions}

The Electric Energy Storage library, which is intended to be included in the Modelica Standard Library, has been described. The structure of the library and key parameters as well as the equations of the models for determining the output variables have been presented. Two typical lab tests for batteries have been simulated as an example to show the interaction of the models and to show the correctness of the model approach.

With the Electric Energy Storage library the user has a powerful tool to cover various applications of energy storages with simulation. In combination with existing libraries, (e.g. the SED) the behavior of electric energy storages and the interaction for example with the pow- 
ertrain of an electric vehicle can be analyzed in detail. Future work will focus on including of thermal models of electirc energy storages and the coupling with the electrical models.

\section{References}

[1] J. V. Gragger, H. Giuliani, C. Kral, T. Bäuml, H. Kapeller, and F. Pirker, "The smartelectricdrives library - powerful models for fast simulations of electric drives," Proceedings of the 5th International Modelica Conference, vol. 2, pp. 571-577, 2006.

[2] Modelica Association, "Modelica - a unified object-oriented language for physical systems modeling, language specification version 3.2," http://www.Modelica.org/, 2010.

[3] Dassault Systems, "Dymola 7.4," http://www.3ds.com/, 2010.

[4] P. Fritzson, Principles of Object-Oriented Modeling and Simulation with Modelica 2.1. IEEE Press, Wiley-Interscience, 2004.

[5] EIG ePLB CO20 lithium ion polymer cell datasheet, EIG, 2010.
[6] A. Jossen, "Fundamentals of battery dynamics," Journal of Power Sources, vol. 154, pp. 530-538, 2006.

[7] S. Buller, "Impedance-based simulation models for energy storage devices in advanced automotive power systems," Aachener Beitraege des ISEA, vol. 31, 2002.

[8] M. Einhorn and J. Fleig, "Improving of active cell balancing by equalizing the cell energy instead of the cell voltage," Proceedings of the 25th International Battery, Hybrid and Fuel Cell Electric Vehicle Symposium and Exposition (EVS25), 2010.

[9] M. Einhorn, F. V. Conte, C. Kral, J. Fleig, and R. Permann, "Parametrization of an electrical battery model for dynamic system simulation in electric vehicles," Proceedings of the IEEE Vehicle Power and Propulsion Conference (VPPC), September 2010.

[10] FTP72 Urban Dynamometer Driving Schedule (UDDS), U.S. Environmental Protection Agency. 